\title{
Measurement of charm production in antineutrino charged-current interactions
}

\author{
CHORUS Collaboration
}

\begin{abstract}
During the years 1994-97, the emulsion target of the CHORUS detector was exposed to the wideband neutrino beam of the CERN SPS collecting about $10^{6}$ neutrino interactions. A measurement of $\bar{\nu}_{\mu}$-induced charm production is performed by using the presence of a $5 \% \bar{\nu}_{\mu}$ component in the $\nu_{\mu}$ beam. The measurement takes advantage of the capability to observe the decay topology in the emulsion. The analysis is based on a sample of charged-current interactions with at least one identified muon. About 100000 were located in the emulsion target and fully reconstructed. By requiring a positive muon charge as determined by the CHORUS muon spectrometer, $32-\bar{\nu}_{\mu}$ induced charm events were observed with an estimated background of 3.2 events. At an average antineutrino energy in the neutrino beam of $18 \mathrm{GeV}$, the charm production rate induced by antineutrinos is measured to be $\sigma\left(\bar{\nu}_{\mu} N \rightarrow \mu^{+} \bar{c} X\right) / \sigma\left(\bar{\nu}_{\mu} N \rightarrow \mu^{+} X\right)=\left(5.0_{-0.9}^{+1.4}\right.$ (stat) \pm 0.7 (syst) $) \%$. The ratio between neutral and charged charm production rates in this process is $\left(2.6_{-1.0}^{+1.7}\right.$ (stat) \pm 0.8 (syst)). The charm production rate as a function of the antineutrino energy is found to be in good agreement with previous results derived from di-lepton data.
\end{abstract}

To be published in Physics Letters B 


\title{
CHORUS Collaboration
}

\author{
G. Önengüt
}

Çukurova University, Adana, Turkey

R. van Dantzig, M. de Jong, O. Melzer, R.G.C. Oldeman ${ }^{1}$, E. Pesen, J.L. Visschers

NIKHEF, Amsterdam, The Netherlands

M. Güler, U. Köse, M. Serin-Zeyrek, R. Sever, P. Tolun, M.T. Zeyrek

METU, Ankara, Turkey

M.G. Catanesi, M. De Serio, M. Ieva, M.T. Muciaccia, E. Radicioni, S. Simone

Università di Bari and INFN, Bari, Italy

A. Bülte, K. Winter

Humboldt Universität, Berlin, Germany ${ }^{2}$

B. Van de Vyver $^{3,4}$, P. Vilain ${ }^{5}$, G. Wilquet ${ }^{5}$

Inter-University Institute for High Energies (ULB-VUB) Brussels, Belgium B. Saitta

Università di Cagliari and INFN, Cagliari, Italy

E. Di Capua

Università di Ferrara and INFN, Ferrara, Italy

S. Ogawa, H. Shibuya

Toho University, Funabashi, Japan

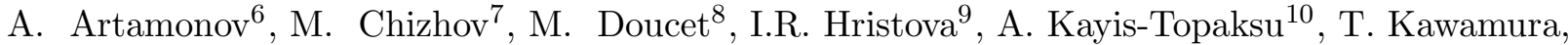
D. Kolev ${ }^{7}$, H. Meinhard, J. Panman, I.M. Papadopoulos, S. Ricciardi ${ }^{11}$, A. Rozanov ${ }^{12}$, R. Tsenov ${ }^{7}$, J.W.E. Uiterwijk, P. Zucchelli ${ }^{3,13}$

CERN, Geneva, Switzerland

J. Goldberg

Technion, Haifa, Israel

M. Chikawa

Kinki University, Higashiosaka, Japan

E. Arik

Bogazici University, Istanbul, Turkey

J.S. Song, C.S. Yoon

Gyeongsang National University, Jinju, Korea

K. Kodama, N. Ushida

Aichi University of Education, Kariya, Japan

S. Aoki, T. Hara

Kobe University, Kobe, Japan

T. Delbar, D. Favart, G. Grégoire, S. Kalinin, I. Makhlioueva

Université Catholique de Louvain, Louvain-la-Neuve, Belgium

P. Gorbunov, V. Khovansky, V. Shamanov, I. Tsukerman

Institute for Theoretical and Experimental Physics, Moscow, Russian Federation N. Bruski, D. Frekers

Westfälische Wilhelms-Universität, Münster, Germany ${ }^{2}$

K. Hoshino, J. Kawada, M. Komatsu, M. Miyanishi, M. Nakamura, T. Nakano, K. Narita, K. Niu,

K. Niwa, N. Nonaka, O. Sato, T. Toshito

Nagoya University, Nagoya, Japan

S. Buontempo, A.G. Cocco, N. D'Ambrosio, G. De Lellis, G. De Rosa, F. Di Capua, A. Ereditato,

G. Fiorillo, A. Marotta, M. Messina, P. Migliozzi, C. Pistillo, L. Scotto Lavina, P. Strolin, V. Tioukov

Università Federico II and INFN, Naples, Italy

K. Nakamura, T. Okusawa 
Osaka City University, Osaka, Japan

U. Dore, P.F. Loverre, L. Ludovici, P. Righini, G. Rosa, R. Santacesaria, A. Satta, F.R. Spada

Università La Sapienza and INFN, Rome, Italy

E. Barbuto, C. Bozza, G. Grella, G. Romano, C. Sirignano, S. Sorrentino

Università di Salerno and INFN, Salerno, Italy

Y. Sato, I. Tezuka

Utsunomiya University, Utsunomiya, Japan

${ }^{1}$ Now at Università La Sapienza, Rome, Italy.

${ }^{2}$ Supported by the German Bundesministerium für Bildung und Forschung under contract numbers $056 \mathrm{BU} 11 \mathrm{P}$ and 05 $7 \mathrm{MS} 12 \mathrm{P}$.

${ }^{3}$ Now at SpinX Technologies, Geneva, Switzerland.

${ }^{4}$ Fonds voor Wetenschappelijk Onderzoek, Belgium.

${ }^{5}$ Fonds National de la Recherche Scientifique, Belgium.

${ }^{6}$ On leave of absence from ITEP, Moscow.

${ }^{7}$ On leave of absence and at St. Kliment Ohridski University of Sofia, Bulgaria.

${ }^{8}$ Now at University of Maryland, MD, USA.

${ }^{9}$ Now at DESY, Hamburg.

${ }^{10}$ On leave of absence from Çukurova University, Adana, Turkey.

${ }^{11}$ Now at Royal Holloway College, University of London, Egham, UK.

${ }^{12}$ Now at CPPM CNRS-IN2P3, Marseille, France.

${ }^{13}$ On leave of absence from INFN, Ferrara, Italy. 


\section{Physics motivation}

Charm production in neutrino and antineutrino charged-current (CC) interactions has been studied by several experiments by looking at the presence of two oppositely charged leptons in the final state. In particular CDHS [1], CCFR [2], CHARM [3], CHARM-II [4], NOMAD [5] and NuTeV [6] have collected a large statistics of opposite-sign dimuon events. The leading muon is interpreted as originating from the neutrino vertex and the other one, of opposite charge, as being the decay product of the charmed particle. In such experiments, the evaluation of the charm production cross-section requires the knowledge of the average muonic branching ratio of the various charmed hadrons, weighted by their relative production rates. Furthermore, experiments of this type suffer from significant background $(\sim 20 \%)$ due to the undetected decay in flight of a pion or a kaon. The background reduction involves cuts on the energies of the primary and decay muons and this makes it difficult to study cross-sections at energies below $20-30 \mathrm{GeV}$.

A different approach using a hybrid emulsion detector was pioneered by the E531 experiment at FNAL [7]. In emulsion, charmed particles can be recognized on the basis of their short flight length and characteristic decay topology. This allowed a study of all decay channels, not only the muonic one, applying loose kinematic cuts, with no required knowledge of branching ratios and with very low background. E531 has measured the charm production rate in CC interactions induced by antineutrinos normalized to the $\bar{\nu}_{\mu} \mathrm{CC}$ sample, although they do not report details on the observation of $\bar{\nu}_{\mu}$ induced charm events. A summary of the available data on antineutrino-induced charm production can be found in Ref. [8]. All together, data at low neutrino energies are limited and no measurement of the production fractions $\bar{f}_{h}$ of the different charm species $h$ exists. With the development of fast automatic emulsion scanning systems in the CHORUS experiment [9], studies of charm production with high statistics become possible. In this paper, the first direct measurements of antineutrinoinduced charm production in emulsion are presented. By using the $\bar{\nu}_{\mu}$ beam contamination and by exploiting the excellent muon charge reconstruction in the spectrometer, CHORUS measured the average production cross-section and its energy dependence as well as the production fractions of neutral and charged charm hadrons.

The comparison of $\bar{\nu}_{\mu}$ and $\nu_{\mu}$ experiments is particularly interesting for the following reasons:

- antineutrino charm production provides information which makes it possible to separate the contribution of valence (absent in the $\bar{\nu}_{\mu}$ case) and sea quarks to the suppression of the charm production rate at low energies.

- in the $\bar{\nu}_{\mu}$ case, charmed antibaryon production is expected to be small, contrary to the $\nu_{\mu}$ case, where charmed baryon production accounts for $\sim 25 \%$ of the total charm production rate [10]. Moreover, quasi-elastic charm production $\left(\nu_{\mu} n \rightarrow \mu^{-} \Lambda_{c}^{+}\right)$, which occurs at a rate of about $15 \%$ of the $\Lambda_{c}^{+}$total production cross-section [10] mainly at low energies, has no equivalent in the $\bar{\nu}_{\mu}$ case.

\section{The experimental set-up}

The CHORUS experiment [9] was designed to investigate neutrino oscillation by searching for the appearance of $\nu_{\tau}$ 's in the SPS wide-band neutrino beam at CERN through the direct observation of the $\tau$ decay in nuclear emulsions. Since charmed particles have a flight length comparable to that of the $\tau$ lepton, the experiment is also suitable for the study of charm production. The detector uses a hybrid approach that combines a nuclear emulsion target with various electronic detectors [9].

The emulsion target, of $770 \mathrm{~kg}$ total mass, is subdivided along the beam axis in four stacks of $1.4 \times 1.4 \mathrm{~m}^{2}$ transverse area. Each stack is laterally divided into eight modules of area $0.36 \times 0.72 \mathrm{~m}^{2}$ comprising 36 plates for a total thickness of $\sim 3 \mathrm{~cm}$. Each plate has a $90 \mu \mathrm{m}$ plastic support coated on both sides by emulsion layers of $350 \mu \mathrm{m}$ thickness. The emulsion target is equipped with a highresolution tracker made out of three interface emulsion sheets and of a set of scintillating fibre tracker planes. The interface emulsion sheets have a plastic base of $800 \mu \mathrm{m}$ coated on both sides with emulsion layers of $100 \mu \mathrm{m}$ thickness. The interface sheets and the fibre trackers provide accurate predictions of 
particle trajectories into the emulsion stack. These predictions are used for the location of the vertex position. The accuracy of the fibre tracker predictions is about $150 \mu \mathrm{m}$ in position and $2 \mathrm{mrad}$ in angle. The emulsion scanning was performed by fully automatic microscopes equipped with a CCD camera and a readout system evolved from the so-called Track Selector [11]. A tracking efficiency of more than $98 \%$ up to 400 mrad angular acceptance is obtained with this method.

The electronic detectors downstream of the emulsion target include a hadron spectrometer which measures the deflection of the charged particles in a hexagonal air-core magnet and a calorimeter where the energy and direction of showers are measured. A muon spectrometer, consisting of six toroidal magnetized iron modules, instrumented with scintillating strips, drift chambers and streamer tubes, determines the charge and momentum of muons and provides a rough measurement of the leakage of hadronic showers behind the downstream surface of the calorimeter. Several planes of scintillator hodoscopes are used for triggering the data acquisition system [12].

\section{$3 \quad$ Data collection}

The CHORUS detector was exposed to the wide-band neutrino beam of the CERN SPS during the years 1994-97, with an integrated flux of $5.06 \times 10^{19}$ protons on target. The beam, of $27 \mathrm{GeV}$ average energy, consists mainly of $\nu_{\mu}$ 's with a $5 \% \bar{\nu}_{\mu}$ component of $18 \mathrm{GeV}$ average energy. Figure 1 shows the energy spectrum of the antineutrino component of the beam as obtained by simulation.

The emulsion scanning of the event starts from the extrapolation to the most downstream interface emulsion sheet of the tracks reconstructed in the fibre tracker. All selected tracks ('scan-back tracks') are searched for in the emulsion interface sheets. The track segments found in these sheets are then used to predict with high precision the position and the angle of the track in the emulsion stack. If found, this track is then followed upstream from plate to plate. In each plate, only the most upstream $100 \mu \mathrm{m}$ layer is scanned searching for a track with the same slope as the one measured in the emulsion interface sheets. The interaction vertex is assumed to be located if the track is not observed in two consecutive plates, the first of which is defined as the vertex plate.

Once the vertex plate has been identified, a very fast scanning system, called 'Ultra Track Selector' (UTS) [13], is used to perform a detailed analysis of the emulsion volume around the vertex position, recording, for each event, all track segments within a given angular acceptance. We refer to this type of scanning, originally developed for the DONUT experiment [14], as NetScan data taking [15]. In CHORUS, the scanning volume is $1.5 \mathrm{~mm}$ wide in each transverse direction and $6.3 \mathrm{~mm}$ along the beam direction, corresponding to eight plates: it contains the vertex plate itself, the plate immediately upstream, acting as a veto for tracks passing through, and the six plates downstream from the vertex plate. The scanning area is centred on the extrapolated transverse position of the scanback track. The angular acceptance corresponds to a cone of $400 \mathrm{mrad}$ half-aperture aligned along the beam direction. At present, about 150000 events have been located in emulsion and analysed with this procedure.

\section{The antineutrino event sample}

The results presented here are based on a sample of 96472 events with at least one reconstructed muon in the spectrometer, successfully extrapolated to the emulsion target and for which the NetScan analysis has been completed. For 2704 of these events, the charge of the muon (or of the highest-energy muon in case of multimuon events), as determined by the muon spectrometer, is found to be positive. To obtain the sample of $\bar{\nu}_{\mu}$ charged-current interactions, one has to subtract from this sample the contaminations due to:

$-\nu_{\mu} \mathrm{CC}$ events with the $\mu^{-}$reconstructed as a $\mu^{+}$;

- $\nu_{\mu}$-induced dimuon events for which the primary muon is not reconstructed while the $\mu^{+}$from the charmed hadron decay is seen;

- punchthrough hadrons traversing the 5.2 interaction lengths of the calorimeter and reconstructed as positive muons in the spectrometer, in CC events with the $\mu$ not identified, or in neutral- 


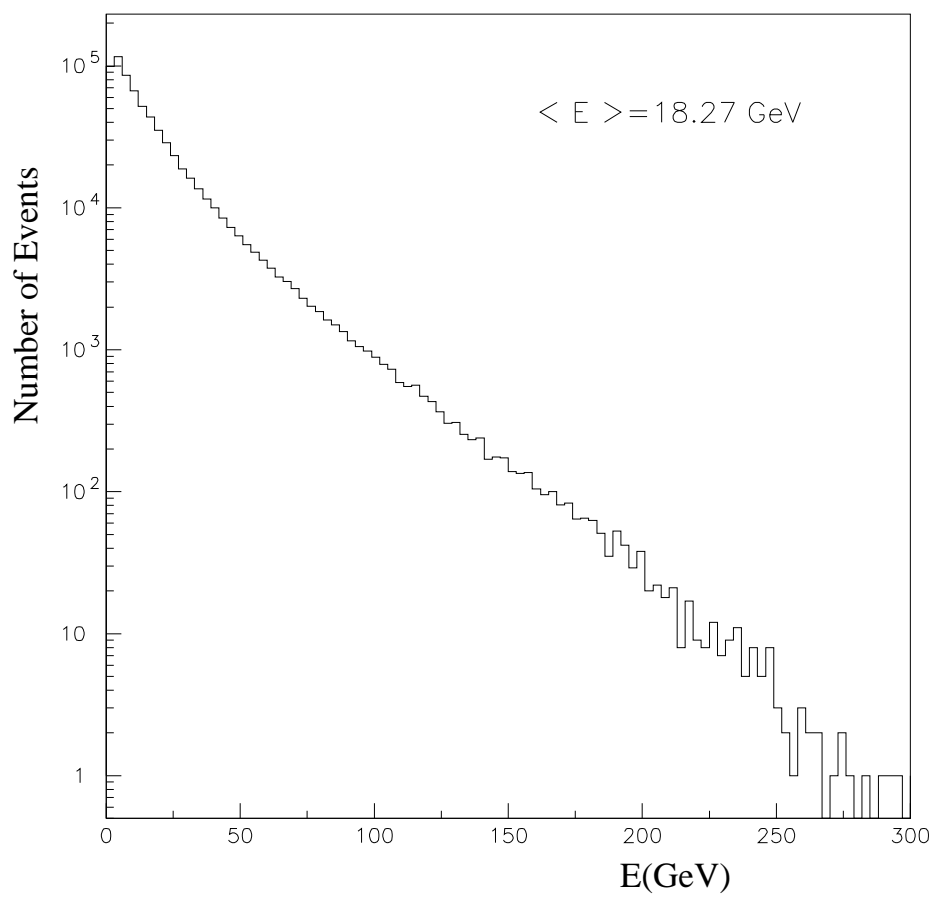

Figure 1: Energy spectrum of the antineutrino component of the beam in the fiducial volume of the CHORUS experiment

Table 1: Estimated contaminations to the sample of $\mu^{+}$located events. The systematic errors take into account the uncertainty on the beam flux measurement [16]

\begin{tabular}{cc}
\hline$\mu^{+}$sample & 2704 events \\
\hline wrong $\mu^{-}$reconstruction & $169 \pm 28$ (stat) \pm 17 (syst) \\
\hline$\mu^{+}$from charm decay & $79 \pm 16$ (stat) \pm 8 (syst) \\
\hline$\pi, K$ punchthroughs & $284 \pm 45$ (stat) \pm 28 (syst) \\
\hline
\end{tabular}

current $(\mathrm{NC})$ interactions.

The contribution from $\pi$ and $\mathrm{K}$ decays into a $\mu$ reconstructed in the spectrometer is negligible. A detailed simulation of the experiment was performed (see Section 6) to evaluate these contributions as well as the overall detection efficiencies. The various contaminations to the sample of $\mu^{+}$located events, shown in Table 1 , have been subtracted statistically to determine the total number of $\bar{\nu}_{\mu} \mathrm{CC}$ interactions. They represent together $(19.7 \pm 2.1) \%$ of the sample of the $\mu^{+}$located events. The mean detection efficiency is found to be $(43.7 \pm 0.6) \%$ for the $\bar{\nu}_{\mu} \mathrm{CC}$ events and $(40.3 \pm 0.4) \%$ for $\nu_{\mu} \mathrm{CC}$ events. It includes the efficiencies of the muon reconstruction in the spectrometer (including charge determination), the tracking downstream to the emulsion stack, and the scanning-back to the vertex plate. The number of $\bar{\nu}_{\mu} \mathrm{CC}$ interactions corrected for the contaminations and for the efficiencies is evaluated to be $4975 \pm 187$ (stat) \pm 53 (syst).

By using the evaluated sample of $\bar{\nu}_{\mu}$ charged-current events, we estimate the ratio of $\bar{\nu}_{\mu} \mathrm{CC}$ and $\nu_{\mu} \mathrm{CC}$ total interaction rates in the CHORUS emulsion target to be $0.021 \pm 0.001$. This is in good agreement with the measurement of $0.022 \pm 0.001$ in the CHORUS calorimeter [16], the correction for the different fiducial volume being small. 


\section{$5 \quad$ Selection of decay topologies}

Track segments collected in the NetScan volume are analysed off-line to reconstruct the complete event topology: segments from different plates are combined into tracks; low-energy tracks are filtered out as well as tracks originating from outside the volume (mainly due to passing-through beam muons); the remaining tracks are assigned to one or several possible vertices and the probability that they match one of the tracks reconstructed in the electronic detectors is evaluated [17]. Events for which the emulsion tracks do not converge to a common vertex potentially contain a short-lived particle decay topology and were selected for further inspection. To select interesting topologies and reduce the amount of fake secondary vertices, the following criteria are applied:

- only tracks reconstructed in more than one plate and with an acceptable straight-line fit are considered;

- one or more emulsion tracks must match the tracks identified as muon in the muon spectrometer; the vertex to which the leading muon track is attached is assumed to be the neutrino interaction vertex;

- at least one emulsion track must exist for which the impact parameter to the reconstructed primary vertex is larger than a cut-off value determined on the basis of the measurement resolution 1);

- the impact parameter must also be smaller than $750 \mu \mathrm{m}$ to reject tracks not related to the neutrino event;

- as the emulsion has no time information, a secondary vertex is validated only if at least one of the tracks attached to it has a good angular matching with a track in the electronic detectors.

These selection criteria were applied to the sample of 2704 located $\mu^{+}$events. A total of 81 events were selected for which a visual inspection was performed to confirm the decay topology. A secondary vertex found in the emulsion is tagged as a decay vertex if no other activity (nuclear break-up or Auger electron) is observed at the decay point and if the number of outgoing tracks is consistent with charge conservation. Moreover, a decay into a single charged particle is accepted only if the angle between the parent and the decay product (kink angle) is greater than $50 \mathrm{mrad}$ and the flight length, i.e. the distance between the primary and decay vertices, is greater than $25 \mu \mathrm{m}$. These requirements ensure a visual inspection efficiency close to $100 \%$. To reduce further the $\nu_{\mu}$ background in the present analysis, the primary vertex, to which the positive muon is attached, is required to be the most upstream one. After visual inspection, 40 events with a positive muon reconstructed in the spectrometer and a topology consistent with a charm decay were identified. The results of the visual inspection are given in Table 2 where the observable decay topologies are classified as even-prong decays V2 or V4 for neutral particles (mainly $\bar{D}^{0}$ ) and odd-prong decays C1, C3 or C5 for charged particles (mainly $D^{-}$, $D_{s}^{-}$), according to the prong multiplicity. The rejected sample consists mainly of neutrino-induced charm events in which the reconstructed positive muon arises from a downstream charm decay vertex, hadronic secondary interactions, delta-rays, or gamma conversions and events with a low-momentum track that, due to multiple scattering, appears to have a large impact parameter with the interaction vertex. The remaining part consists of fake vertices due to imperfections of the reconstruction program.

\section{$6 \quad$ Reconstruction efficiency and background evaluation}

Detection efficiencies and backgrounds were evaluated with a detailed simulation of the detector response with a GEANT3 [18] based program called EFICASS. Large samples of deep-inelastic neutrino interactions (DIS) were generated according to the beam spectrum by the CHORUS JETTA [19] generator based on LEPTO [20] and JETSET [21]. Quasi-elastic (QE) interactions and resonance production events were generated with the RESQUE [22] package with a rate of $9.6 \%$ relative to

\footnotetext{
1) The impact parameter is required to be greater than $\sqrt{\left(5.0^{2}+(2 d x \times \sigma)^{2}\right)} \mu \mathrm{m} ; \sigma=\sqrt{\left(0.003^{2}+(0.0194 \times \theta)^{2}\right)}$ is a parametrization of the angular error and $d x$ is the distance of the vertex from the most upstream daughter track segment.
} 
Table 2: Results of the visual inspection (eye-scan) of charm candidates

\begin{tabular}{cc}
\hline Secondary vertex topology & Number of events \\
\hline V2 & 18 \\
V4 & 7 \\
C1 & 6 \\
C3 & 7 \\
C5 & 2 \\
\hline
\end{tabular}

Table 3: Detection efficiencies for $\bar{\nu}_{\mu}$ and $\nu_{\mu}$ induced charm events in emulsion with at least one muon reconstructed in the spectrometer. The meaning of $\epsilon_{\text {det }}$ and $\epsilon_{\text {sel }}$ is explained in the text

\begin{tabular}{ccc}
\hline & $\epsilon_{\text {det }}(\%)$ & $\epsilon_{\text {sel }}(\%)$ \\
\hline$\nu_{\mu}$ CC charm & $32.2 \pm 0.2$ & $28.6 \pm 0.2$ \\
\hline $\bar{\nu}_{\mu}$ CC charm & $28.2 \pm 1.0$ & $26.5 \pm 1.0$ \\
\hline
\end{tabular}

deep-inelastic scattering reactions in the neutrino case and of $26 \%$ in the antineutrino case. The simulated response of the electronic detectors was processed through the same reconstruction program, CHANT, as used for the experimental data. Similarly, the efficiency of the vertex location procedure described in Section 3 was obtained by a realistic simulation of the grains produced in emulsion along the scanned-back track. The procedure simulates the known performance of the Track Selector. The combined average efficiencies, $\epsilon_{\text {det }}$, to reconstruct the muon in the spectrometer, perform the tracking downstream to the emulsion stack and find the interaction vertex are shown in Table 3 for $\nu_{\mu}$ and $\bar{\nu}_{\mu}$ charm events. These efficiencies are lower than those for CC events reported in Section 4. This can be understood qualitatively by two effects: the higher average track multiplicity in charm events makes the track reconstruction in the target tracker more difficult and, specially for $\bar{\nu}_{\mu}$ events, the momentum and angular distributions of the primary muons are different in charm and non-charm events.

The efficiency of the NetScan procedure represents the probability to reconstruct the vertex and the decay topology of the charmed particle in the scanned emulsion volume. To evaluate the efficiency, the emulsion data of the simulated events were merged with real NetScan data where the volume did not contain any reconstructed vertex but only tracks which stop or pass through the volume, thus representing realistic background conditions. The performance of the automatic scanning system in terms of tracking efficiency and angular resolution was simulated by using data accumulated in the NetScan procedure. The NetScan efficiencies, containing both the geometrical acceptance and the reconstruction efficiency, are shown in Table 4 and in Table 5 for $\nu_{\mu}$ and $\bar{\nu}_{\mu}$ events and for each decay topology. The contribution from charmed $D^{ \pm}$and $D_{s}^{ \pm}$mesons was evaluated assuming $D_{s}^{+} / D^{+}=0.627 \pm 0.073$ [23] and the production ratio as obtained in [24, 25]. The errors include the effect of Monte Carlo statistics used for the efficiency evaluation, instrumental effects, and the uncertainty on $D_{s}^{+} / D^{+}$. For the $\bar{\nu}_{\mu}$ sample, the contribution of $\bar{\Lambda}_{c}$ was neglected.

The most important potential source of background on the sample of antineutrino charm can-

Table 4: NetScan efficiency containing geometrical acceptance and reconstruction efficiency for decays of neutral charmed hadrons into two and four prongs, respectively for $\bar{\nu}_{\mu}$ and $\nu_{\mu}$-induced CC events

\begin{tabular}{ccc}
\hline & $\bar{\nu}_{\mu}$ & $\nu_{\mu}$ \\
\hline & $\bar{D}^{0}$ & $D^{0}$ \\
\hline$\epsilon_{2 p}(\%)$ & $53.7 \pm 2.5$ & $56.5 \pm 0.5$ \\
\hline$\epsilon_{4 p}(\%)$ & $68.4 \pm 4.7$ & $74.7 \pm 0.9$ \\
\hline
\end{tabular}


Table 5: NetScan efficiency containing geometrical acceptance and reconstruction efficiency for decays of charged charmed hadrons into one, three and five prongs, respectively for $\bar{\nu}_{\mu}$ and $\nu_{\mu}$-induced CC events

\begin{tabular}{cc|cc}
\hline & $\bar{\nu}_{\mu}$ & \multicolumn{2}{|c}{$\nu_{\mu}$} \\
\hline & $D^{-}+D_{s}^{-}$ & $D^{+}+D_{s}^{+}$ & $\Lambda_{c}^{+}$ \\
\hline$\epsilon_{1 p}(\%)$ & $31.0 \pm 5.2$ & $27.1 \pm 1.0$ & $19.9 \pm 1.6$ \\
\hline$\epsilon_{3 p}(\%)$ & $56.3 \pm 5.0$ & $60.4 \pm 1.3$ & $46.3 \pm 2.0$ \\
\hline$\epsilon_{5 p}(\%)$ & $60.5 \pm 24.4$ & $72.3 \pm 3.5$ & $48.7 \pm 4.5$ \\
\hline
\end{tabular}

didates is due to $\nu_{\mu}$-induced charm events for which either the primary $\mu^{-}$charge is reconstructed as positive or the $\mu^{+}$track from a muonic charm decay is seen in the spectrometer but is wrongly matched to an emulsion track at the primary vertex. To reduce this background, a set of selection criteria improving the reliability of the muon reconstruction is applied to the events with an observed decay topology:

- Low momentum muons stopping inside the spectrometer, for which the reconstruction is based on few hits in the electronic detectors, are discarded.

- The distance of the impact point on the spectrometer entrance surface from its axis is required to be smaller than $150 \mathrm{~cm}$, ensuring a long potential path through the toroidal magnets.

- Several muon reconstruction algorithms in the spectrometer are available, using only hits in the drift chambers or the combined data from all electronic detectors. Consistency checks are applied when a muon track is reconstructed by more than one algorithm. Otherwise, the track length is required to be greater than three spectrometer gaps (two magnets).

- When matching a spectrometer track with a track in the fibre trackers, a tighter cut is applied on the $\chi^{2}$ of the global track fit.

With the above muon reconstruction criteria, the probability for a $\nu_{\mu}$-induced charm event to be selected as a $\bar{\nu}_{\mu}$-charm candidate is reduced from $(0.12 \pm 0.02) \%$ to $(0.02 \pm 0.01) \%$ while the combined average efficiencies, $\epsilon_{\text {sel }}$, defined as $\epsilon_{\text {det }}$ but after selection, are only slightly affected. They are given in the second column of Table 3 for $\nu_{\mu}$ and $\bar{\nu}_{\mu}$ charm events. The dependence of $\epsilon_{\text {det }}$ and $\epsilon_{\text {sel }}$ on the (anti)neutrino energy is shown in Figure 2 for charm events induced by $\bar{\nu}_{\mu}$ and $\nu_{\mu}$. Taking into account the efficiency to detect the charm decay in these interactions, the number of background events from this source is estimated to be $1.23 \pm 0.25$.

The other significant sources of background are:

- Hadronic white kinks (WK). These are hadronic nuclear interactions with no heavily ionizing tracks or other evidence for nuclear break-up (blobs or Auger electrons), that mimic a decay kink topology. An interaction length of $2.5 \pm 0.5 \mathrm{~m}$ for this process was obtained from Monte Carlo simulation [26] leading to an expected number of WK events in the final C1 sample of $0.8 \pm 0.1$.

- Strange particles can fake a charm topology although they have much longer mean decay length. The background in the V2 sample from $K_{s}^{0}$ and $\Lambda$ decays is evaluated to be $0.9 \pm 0.1$ events. The expected background in the $\mathrm{C} 1$ sample from the decay of charged strange particles is $0.22 \pm 0.09$ events. No strong energy dependence of these backgrounds is observed.

Other background processes which may fake three or more prong decays were investigated but were found to be negligible. The total number of background events is thus estimated to be $3.2 \pm 0.3$.

\section{$7 \quad$ Results}

Table 6 shows the decay topologies of the 32 events surviving the cuts on muon reconstruction, the estimated residual background for each topology, and the number of events after background subtraction, weighted with the inverse of the NetScan efficiency. Taking into account the measured 

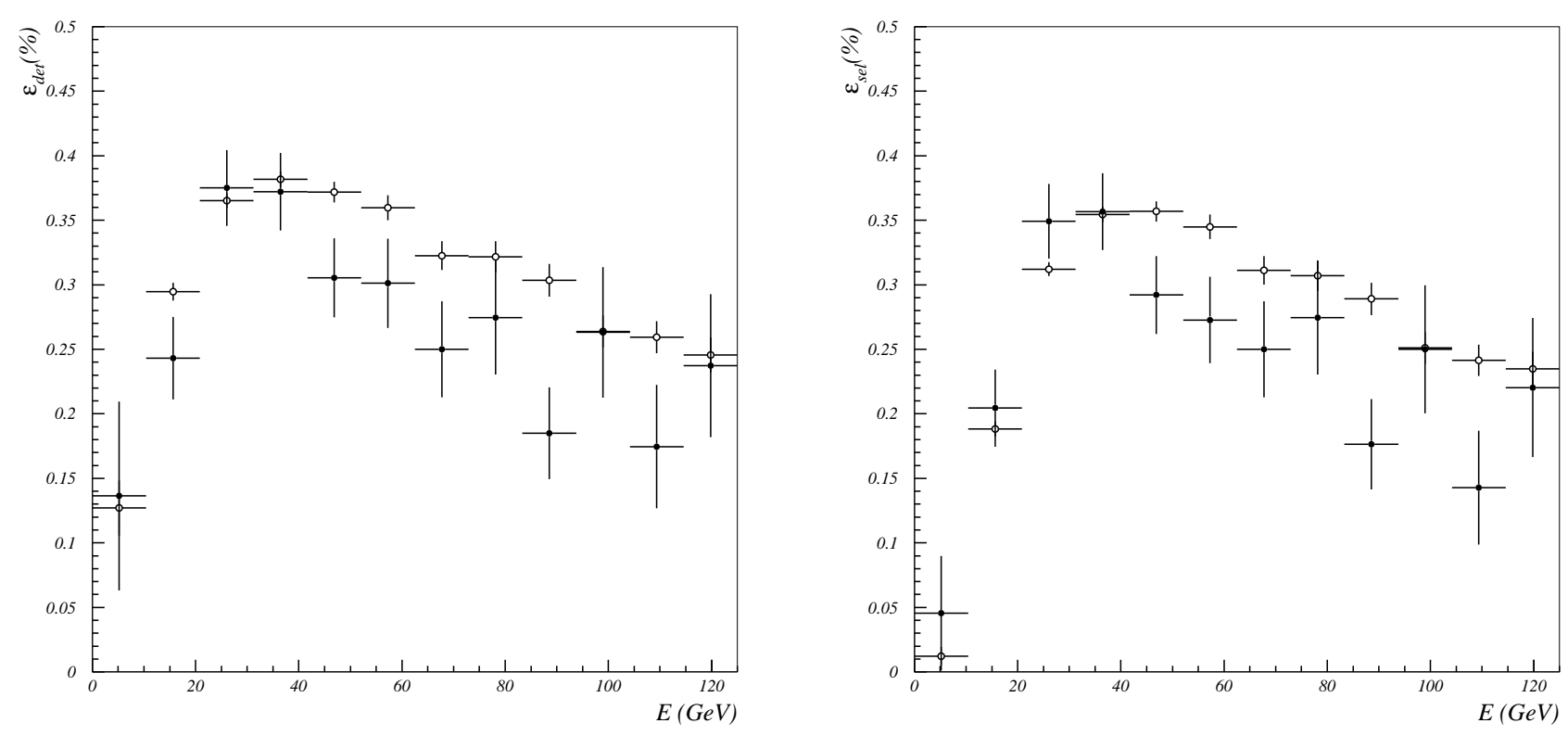

Figure 2: Reconstruction efficiency (including muon reconstruction and charge determination) for $\bar{\nu}_{\mu}$ CC charm (full dots) and $\nu_{\mu}$ CC charm (open circles) events as a function of true (anti)neutrino energy (left panel) and selection criteria efficiency (right panel)

Table 6: Candidate events and estimated backgrounds for each decay topology after applying the cut on muon reconstruction. The last column gives the final sample of events after background subtraction and NetScan efficiency correction

\begin{tabular}{cccc}
\hline Decay topology & Events & Background & Weighted sample \\
\hline V2 & 16 & $1.4 \pm 0.2$ & $27.2_{-6.8}^{+8.9}$ (stat) $\pm 1.3($ syst $)$ \\
V4 & 6 & $0.13 \pm 0.05$ & $8.6_{-3.1}^{+4.8}$ (stat) \pm 0.6 (syst) \\
C1 & 4 & $1.3 \pm 0.2$ & $8.6_{-5.1}^{+9.1}$ (stat) $\pm 1.4($ syst $)$ \\
C3 & 4 & $0.3 \pm 0.1$ & $6.5_{-2.9}^{+5.0}$ (stat) \pm 0.6 (syst) \\
C5 & 2 & $0.02 \pm 0.01$ & $3.3_{-2.1}^{+3.8}$ (stat) \pm 1.3 (syst) \\
\hline
\end{tabular}

branching ratio of $\bar{D}^{0}$ into all neutrals of about $25 \%$ [27] as well as the detection efficiencies described in Section 6, a value of

$$
\left.\sigma\left(\bar{\nu}_{\mu} N \rightarrow \mu^{+} \bar{c} X\right) / \sigma\left(\bar{\nu}_{\mu} N \rightarrow \mu^{+} X\right)=\left(5.0_{-0.9}^{+1.4} \text { (stat) } \pm 0.7 \text { (syst }\right)\right) \%
$$

is obtained for the charm production rate in charged-current interactions induced by $\bar{\nu}_{\mu}$ normalized to the $\bar{\nu}_{\mu} \mathrm{CC}$ sample. This has to be compared with $(6.1 \pm 0.6) \%$ as evaluated in neutrino interactions $[24,25]$. This result is consistent with a reduced production of charmed baryons in the $\bar{\nu}_{\mu}$ case. The statistical error was derived by using the $68.27 \%(1 \sigma)$ confidence interval in the unified approach for the analysis of small signals in presence of background [28]. The systematic error includes the effect of Monte Carlo statistics used for the efficiency evaluation, instrumental effects, the uncertainty on the ratio $D_{s}^{+} / D^{+}$, the branching ratio of $\bar{D}^{0}$ into neutrals, and on the rate of the $\bar{\Lambda}_{c}$ production.

Figure 3 displays the above cross-section ratio as a function of the antineutrino energy (below 100 $\mathrm{GeV}$ ), together with the corresponding results derived from di-lepton data [8] and with the theoretical prediction obtained from a leading-order calculation with $m_{c}=1.31 \mathrm{GeV} / c^{2}$ [30]. The comparison 


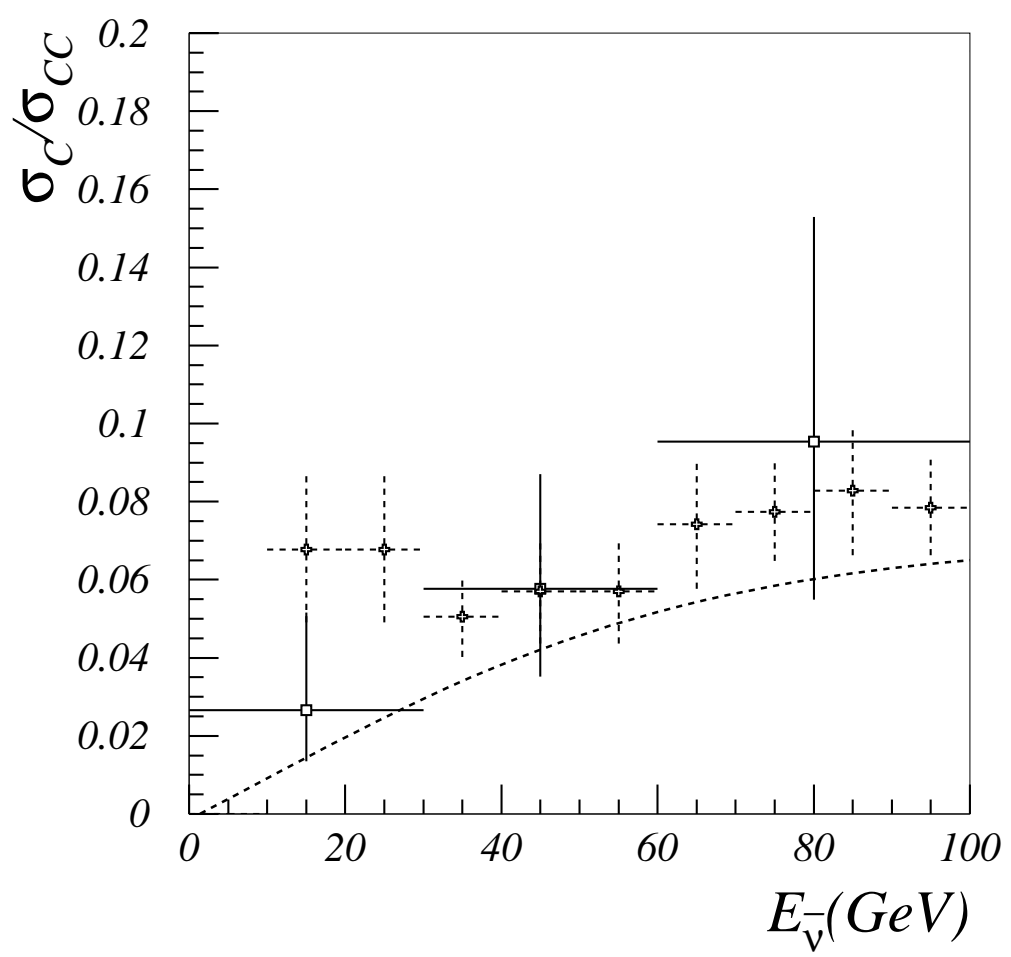

Figure 3: Charm production rate in $\bar{\nu}_{\mu} \mathrm{CC}$ interactions as a function of the antineutrino energy: measured in CHORUS (empty box) and derived from di-lepton data (dashed lines) [8] using the muonic branching ratio $B_{\mu}$ given by Ref. [29]. The line shows the theoretical prediction obtained from a leading-order calculation with $m_{c}=1.31 \mathrm{GeV} / c^{2}[30]$

shows a good agreement with di-lepton data at high energy, whereas CHORUS measurements give a lower production rate at low antineutrino energy, as expected from the absence of quasi-elastic charm production. The theoretical prediction shows a satisfactory agreement with CHORUS antineutrino data at low energy.

We also measured the ratio of the production fractions of neutral and charged charmed hadrons to be $2.6_{-1.0}^{+1.7}$ (stat) \pm 0.8 (syst). This result has to be compared with a value of about 1.0 obtained in the neutrino case [24, 25], supporting the expectation that the charmed baryon production is strongly suppressed in antineutrino interactions.

\section{Acknowledgements}

We gratefully acknowledge the help and support of the neutrino-beam staff and of the numerous technical collaborators who contributed to the detector construction, operation, emulsion pouring, development, and scanning. The experiment was made possible by grants from the Institut Interuniversitaire des Sciences Nucléaires and the Interuniversitair Instituut voor Kernwetenschappen (Belgium); the Israel Science Foundation (Grant 328/94) and the Technion Vice President Fund for the Promotion of Research (Israel); CERN (Geneva, Switzerland); the German Bundesministerium für Bildung und Forschung (Germany); the Institute of Theoretical and Experimental Physics (Moscow, Russia); the Istituto Nazionale di Fisica Nucleare (Italy); the Promotion and Mutual Aid Corporation for Private Schools of Japan and Japan Society for the Promotion of Science (Japan); Korea Research Founda- 
tion Grant (KRF-2003-005-C00014) (Republic of Korea); the Foundation for Fundamental Research on Matter FOM and the National Scientific Research Organization NWO (The Netherlands); and the Scientific, and Technical Research Council of Turkey (Turkey). We gratefully acknowledge their support.

\section{References}

[1] H. Abramowicz et al., CDHS Collaboration, Z. Phys. C15 (1982) 19.

[2] S.A. Rabinowitz et al., CCFR Collaboration, Phys. Rev. Lett. 70 (1993) 134.

[3] M. Jonker et al., CHARM Collaboration, Phys. Lett. B107 (1981) 241.

[4] P. Vilain et al., CHARM II Collaboration, Eur. Phys. J. C11 (1999) 19.

[5] P. Astier et al., NOMAD Collaboration, Phys. Lett. B486 (2000) 35.

[6] M. Goncharov et al., NuTeV Collaboration, Phys. Rev. D64 (2001) 112006.

[7] N. Ushida, et al., E531 Collaboration, Phys. Lett. B206 (1988) 375.

[8] G. De Lellis, et al., J. Phys. G28 (2002) 1515.

[9] E. Eskut, et al., CHORUS Collaboration, Nucl. Instrum. and Methods A401 (1997) 7.

[10] A. Kayis-Topaksu et al., CHORUS Collaboration, Phys. Lett. B575(2003) 198.

[11] T. Nakano. Ph.D. thesis, Nagoya University, Japan, 1997.

[12] M.G. van Beuzekom, et al., CHORUS Collaboration, Nucl. Instrum. and Methods A427 (1999) 587.

[13] T. Nakano, Proc. Intern. Europhysics Conf. on High Energy Physics, Budapest, Hungary (2001).

[14] K. Kodama, et al., DONUT Collaboration, Nucl. Instrum. and Methods A493 (2002) 45.

[15] N. Nonaka, Ph.D. thesis, Nagoya University, Japan (2002).

[16] R.G.C. Oldeman, Ph.D. thesis, University of Amsterdam, The Netherlands, (June 2000).

[17] A.M. Güler, Ph.D. thesis, Middle East Technical University, Ankara, Turkey (2000).

[18] GEANT 3.21, CERN program library long write up W5013.

[19] P. Zucchelli, Ph.D. thesis, Universitá di Ferrara, Italy (1995).

[20] G. Ingelman, Preprint TSL/ISV 92-0065, Uppsala University, Sweden (1992).

[21] T. Sjöstrand, Comput. Phys. Commun. 82 (1994) 74.

[22] S. Ricciardi, Ph.D. thesis, Universitá di Ferrara, Italy (1996).

[23] G. De Lellis et al., Phys. Lett. B550 (2002) 16.

[24] A. Kayis-Topaksu et al., CHORUS Collaboration, Phys. Lett. B527 (2002) 173.

[25] A. Kayis-Topaksu et al., CHORUS Collaboration, Phys. Lett. B555 (2003) 156.

[26] A. Satta, Ph.D. thesis, Universitá La Sapienza di Roma, Italy (2001).

[27] CHORUS Collaboration, "Measurement of $\mathrm{D}^{0}$ production and decay in neutrino interactions", in preparation.

[28] G.J. Feldman and R.D. Cousins, Phys. Rev. D57 (1998) 3873.

[29] A. Kayis-Topaksu et al., CHORUS Collaboration, Phys. Lett. B549 (2002) 48.

[30] J. M. Conrad, M. H. Shaevitz and T. Bolton, Rev. Mod. Phys. 70 (1998) 1341. 